\title{
The duration of recessions follows an exponential not a power law
}

\author{
Ian Wright \\ iKuni Inc., \\ 3400 Hillview Avenue, Building 5, Palo Alto, CA 94304, USA \\ Fax: +16503209827 \\ Phone: +16503205355
}

\begin{abstract}
Ormerod and Mounfield [1] analyse GDP data of 17 leading capitalist economies from 1870 to 1994 and conclude that the frequency of the duration of recessions is consistent with a power-law. But in fact the data is consistent with an exponential (Boltzmann-Gibbs) law.
\end{abstract}

Key words: Econophysics, Power-laws, Exponential-laws PACS: 64.60.Lx, 64.60.Ht

Ormerod and Mounfield [1] analyse GDP data of 17 leading capitalist economies from 1870 to 1994 and measure the frequency of duration of recessions. The duration of a recession is defined as the number of years in which real GDP growth (measured as a percentage change) is less than zero. Table 1 reproduces their data.

Email address: wright@ikuni.com (Ian Wright).

$U R L$ : ianusa.home.mindspring. com (Ian Wright). 
Table 1

Duration of recessions

\begin{tabular}{llllllll}
\hline Duration (yr) & 1 & 2 & 3 & 4 & 5 & 6 & 7 \\
\hline Frequency & 206 & 88 & 23 & 10 & 5 & 3 & 1
\end{tabular}

The authors propose that the relationship between the frequency, $N$, of observations and the duration, $D$, in years of a recession is a power-law

$$
N=\frac{\alpha}{D^{\beta}}
$$

The authors use a non-linear algorithm to determine the best-fit parameters $\alpha=209.6$ and $\beta=1.69$. A non-linear fit using the Levenberg-Marquardt method reproduces these results, yielding parameters $\alpha=209.57$ and $\beta=$ 1.694 (2 d.p.), with root mean square (RMS) error 11.86. The authors note, however, that an 'essential problem of over-prediction of the tail of the distribution was not solved'.

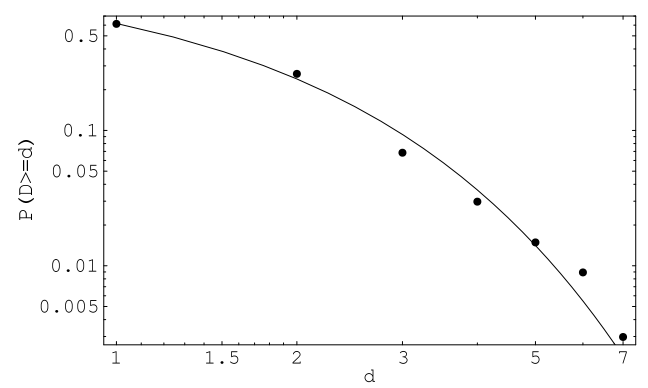

(a) The solid line is an exponential fit, $f(D)=\mu \lambda \mathrm{e}^{-\lambda D}$, to the data.

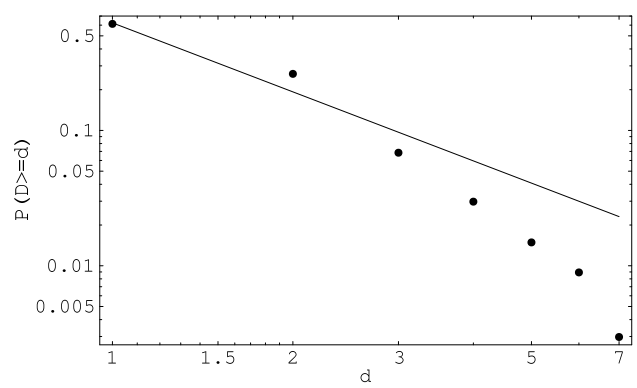

(b) The solid line is a power-law fit, $f(D)=\frac{\alpha}{D^{\beta}}$, to the data.

Fig. 1. Graphical analysis of the frequency of duration of recessions plotted as a cumulative probability distribution in log-log scale.

The problem of over-prediction of the tail of the distribution is solved if the relationship between $N$ and $D$ is considered to be an exponential law

$$
N=\mu \lambda \mathrm{e}^{-\lambda D}
$$

A non-linear fit yields parameters $\mu=564.85$ and $\lambda=0.94$, with RMS error 4.67. Figure 1 plots both the exponential and power-law fits to the data as complementary cumulative distribution functions (ccdf) in log-log scale. The data is clearly consistent with an exponential not a power law 1

1 The frequency of the size of recessions, defined as the cumulative fall in output that occured during the recession, is also analysed by the authors. They conclude that this data follows a power-law. But in this case also an exponential law yields a lower RMS error than a power-law. 
The authors note that if durations of one year are excluded from the data (representing $61 \%$ of the data) then the power-law fit improves. In this case, the exponential fit has a RMS error of 2.01, whereas a power-law fit has a RMS error 0.57. Figure 2 plots the exponential and power-law fits to the reduced data. The reduced data is clearly consistent with a power-law not an exponential law.

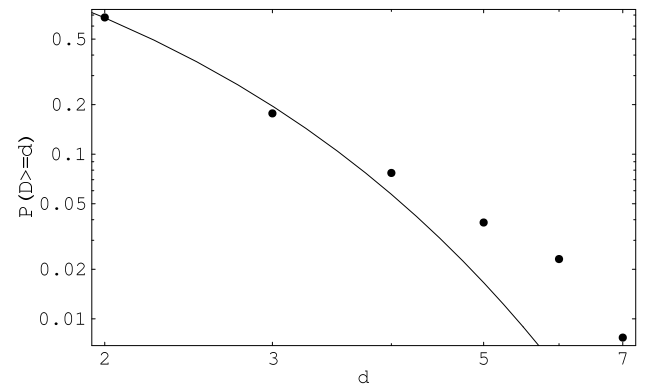

(a) The solid line is an exponential fit, $f(D)=\mu \lambda \mathrm{e}^{-\lambda D}$, to the data.

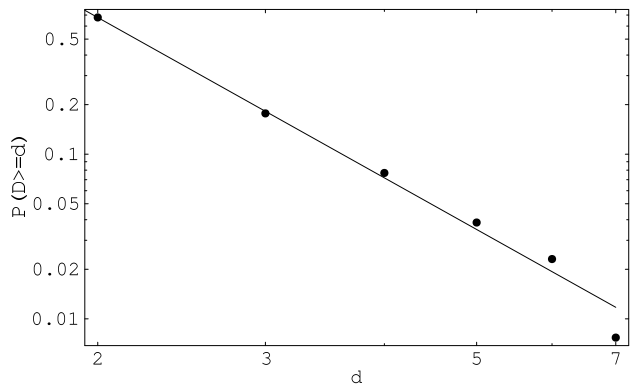

(b) The solid line is a power-law fit, $f(D)=\frac{\alpha}{D^{\beta}}$, to the data.

Fig. 2. Graphical analysis of the frequency of duration of recessions that last two years or more plotted as a cumulative probability distribution in log-log scale.

The authors propose that economic management often prevents recessions lasting more than one year, but if they do last longer, then subjective expectations of growth become depressed and recessions may then occur on all scales of duration, resulting in a power-law. This explanation is indeed consistent with the data once recessions lasting one year are dropped. But the explanation is redundant once all the data is correctly classified as following an exponential distribution.

\section{References}

[1] P. Ormerod and C. Mounfield, Power law distribution of the duration and magnitude of recessions in capitalist economies: breakdown of scaling, Physica A 293 (2001) 573-582. 